\title{
Induced abortion did not increase the risk of breast cancer
}

\author{
Melbye M, Wohlfahrt J, Olsen JH, et al. Induced abortion and the risk of breast cancer. N Engl J Med 1997 Jan 9;336:81-5.
}

\section{Objective}

To determine whether an association exists between induced abortion and the risk of breast cancer.

\section{Design}

Cohort study of women followed up from 1968-92.

\section{Setting}

Population based study in Denmark.

\section{Participants}

All Danish women born from 1 April 1935 to 31 March 1978. In total, 1529512 women were included in the cohort.

\section{Assessment of risk factors}

Through a linkage with the National Registry of Induced Abortions, information on the number and dates of induced abortions was combined with information on the gestational age of each aborted fetus. The induced abortions included in this analysis were those that occurred between 1973 and 1992. Data on the obstetric history (age, parity, age at delivery of first child) were taken from the cohort database.

\section{Main outcome measures}

New cases of breast cancer identified through a linkage with the Danish Cancer Registry. Follow up for breast cancer began on 1 April 1968 or on the woman's 12th birthday, whichever came later.

\section{Main results}

Of the 1529512 women in the cohort, 280965 had a total of 370715 induced abortions and 10246 developed breast cancer. After adjustment for age, parity, age at delivery of first child, and calendar period, the overall risk of breast cancer in women with a history of induced abortion did not differ from that of women without such a history (relative risk [RR] 1.00, 95\% CI 0.94 to 1.06). No increases in the risk of breast cancer were found in subgroups of women defined by age at induced abortion, parity, number of abortions, time since abortion, or age at diagnosis of breast cancer. The RR of breast cancer did increase with increasing gestational age of the fetus. With each 1 week increase in gestational age, a 3\% increase occurred in the risk of breast cancer. The RR increased from 0.81 (CI 0.58 to 1.13 ) among women whose most recent induced abortion was at $<7$ weeks of gestation to 1.38 (CI 1.00 to 1.90) among women whose most recent abortion was at $>12$ weeks gestation (reference category, induced abortion at 9-10 weeks gestation).

\section{Conclusion}

History of induced abortion did not increase the risk of breast cancer overall, but an increased risk was identified after second trimester abortions.

\section{Sources of funding:Danish Cancer Society; Danish National Research Foundation; U.S. Department of Defense.}

For article reprint: Dr M Melbye, Department of Epidemiology Research, Danish Epidemiology Science Center, Statens Serum Institut, 5 Artillerivej, DK-2300 Copenhagen S, Denmark. Fax +4532683165

Adapted from an abstract published in ACP Journal Club 1997 Jul-Aug;127:18

\section{Commentary}

The study by Melbye $e t$ al is a useful addition to sexual and reproductive health care because it clarifies one of the many grey areas in our knowledge. Abortion and breast cancer are emotional subjects, and making the decision to have an abortion is difficult enough without having to consider the possible future risk of developing breast cancer. When a link was hypothesised in $1980,{ }^{1}$ it produced another area of concern for those involved in providing health care to women. Other studies have shown conflicting results, ${ }^{2}$ but most depended on reporting of abortions by women themselves, which is known to be unreliable. ${ }^{3}$ The strengths of this study are in its sheer size, prospective nature, and non-dependence on self reporting of abortion.

For nurses working in family planning practice, many issues need to be discussed so that women can make informed choices about contraceptive or pregnancy care. When a theoretical or uncertain risk is identified, it can make discussion of risks and benefits difficult-women like to be told what their real risks are. This latest research provides clear evidence that nurses can reassure women who require abortion that their overall risk of breast cancer is not increased. The findings also support the recommendation that abortion is preferable in the first trimester.

Shelley Mehigan, RGN, OHNC Clinical Nurse Specialist Chair, Family Planning Forum Royal College of Nursing Maidenhead, UK

1 Russo J, Russo IH. Susceptibility of the mammary gland to carcinogenesis. II. Pregnancy interruption as a risk factor in tumour incidence. Am J Pathol 1980;100:497-512.

2 Brind J, Chinchilli VM, Severs WB, et al. Induced abortion as an independent risk factor for breast cancer: a comprehensive review and meta-analysis. I Epidemiol Community Health 1996;50:481-96.

3 Jones EF, Darroch Forrest J. Underreporting of abortion in surveys of US women: 1976 to 1988. Demography 1992;29:113-26. 